$\mathrm{ADHD}$ is linked to deprivation, but there are not enough studies in literature to prove or disprove this assumption. The aim of this study was to investigate the relationship between social deprivation and ADHD.

Method We included all patients diagnosed with ADHD by the community paediatric department (only those on medications). Postcodes of these patients were used to produce deprivation scores, which included overall deprivation and sub-scores for income, social and housing factors. Indices of Deprivation 2010 are available for 32,482 small geographical areas (Lower Super Output Areas, LSOAs) in England, ranked from 1 (most deprived) to 32,482 (least deprived). These are further divided into fifths to produce English deprivation quintiles. Each postcode was then allocated to a quintile based on their deprivation score, where quintile 1 represents the most deprived.

Results A total of 144 patients diagnosed with ADHD were being treated with medication. The male to female ratio was 4.5:1 (M: F). The deprivation scores were calculated and it showed that 64 patients (44.4\%) were in the most deprived quintile (quintile 1), and followed in a relatively linear pattern. A similar pattern was seen for income, crime, employment, education, skills and training domain and health deprivation and disability, where $69,57,74,69$ and 59 patients were placed in quintile 1 , respectively.

Conclusion Our study shows an association between the prevalence of $\mathrm{ADHD}$ in children and deprivation index. Also there is clear link between sub scores for income, crime, employment, education, skills and training domain and health deprivation and disability and prevalence of $\mathrm{ADHD}$.

Indices of deprivation could be used to predict the expected prevalence of ADHD within the community and thus plan allocation of resources. Ours is a small sample size, but results support further investigation with a larger study.

\section{G208 DISTINCT HEALTHCARE PRIORITIES IN EARLY ADOLESCENCE}

doi:10.1136/archdischild-2013-304107.220

'RJ Bensted, ${ }^{2} \mathrm{DS}$ Hargreaves, ${ }^{3} \mathrm{~J}$ Lombard, ${ }^{4} \mathrm{U}$ Kilkelly, ${ }^{5} \mathrm{RM}$ Viner. ${ }^{1}$ Endocrinology, Barts and the London NHS Trust, London, UK; ' Institute of Child Health, UCL, London, UK; ${ }^{3}$ University College Cork, Cork, Ireland; ${ }^{4}$ University College Cork, Cork, Ireland; 5 Institute of Child Health, UCL, London, UK

Aims To investigate differences in healthcare priorities between children ( $<12$ years), adolescents ( $13-15$ years) and young adults (16-18 years).

Methods Data were analysed from the 2010 Council of Europe survey into child-friendly healthcare. 2023 respondents under the age of 18 from 8 European countries rated the importance of 9 healthcare factors.

Having your parent/family with you

Knowing the name of the doctor/nurse

Having treatment explained in advance/being prepared

Being able to understand what the doctor is saying

Being able to ask questions

Being listened to

Not being afraid

Not being in pain

Not feeling rushed

The relative importance of items was compared within each age group using mean score differences and between age groups using logistic regression.

Results All age groups rated being listened to as the most important item. Children rated being with parents more important than understanding the doctor (mean difference $(\mathrm{x})=0.47$ (95\% Confidence Interval $0.21, .72)$ ). This finding was reversed among adolescents $(x=-0.74(-0.97,-0.50))$ and young adults $(x=-1.67(-1.95$,
-1.38)). Similarly, being with parents was rated more important than being able to ask questions for children $(x=0.91(.62,1.21))$ but the reverse was found for adolescents $(x=0.36(-0.62,0.11))$ and young adults $(\mathrm{x}=-1.44(-1.75,-1.14))$.

Among children, pain control scored higher than understanding doctors $(\mathrm{x}=0.31(.07,0.54))$ and asking questions $(\mathrm{x}=0.80(.51$, $1.08))$. There was no significant difference between these items for adolescents $(\mathrm{x}=-0.09(-0.32,0.13))$ and $\mathrm{x}=0.24(.00,0.48))$ respectively. Among young adults, pain control was less important than understanding doctors $(x=-0.38(-0.61,-0.14))$ and equally important to asking questions $(\mathrm{x}=-0.22(-0.48,0.03))$.

The changes in relative priorities between childhood and adolescence remained significant in the logistic regression models after adjusting for sex, long standing illness and nationality (all $\mathrm{p}<0.001$ ).

Conclusion Healthcare priorities differ significantly between childhood and early adolescence. However, being listened to was rated the most important priority at all ages.

\section{G209 MANAGING SUBSTANCE MISUSE IN YOUNG PEOPLE - WHAT WORKS?}

doi:10.1136/archdischild-2013-304107.221

J Beckmann. Northwick Park Hospital, North West London Hospitals NHS Trust, London, UK

Alcohol and Drug use in Young People is a current national topic of great curiosity which occasionally, junior doctors manage with less interest during busy $\mathrm{A} \& \mathrm{E}$ shifts and pressing admission beds. A taboo subject infrequently discussed in the Paediatric/Adolescent patient history, health professional competence in assessing risk may also be variable, but could be improved by a strong presence of multi-departmental teaching, publicity of accessible intranet management guidance and on-site service information-specific patient and parent leaflets.

A retrospective analysis of attendance data for 9-17 year-olds to a busy District General Hospital Accident and Emergency Department during a peak festive and school holiday season was conducted. Young persons presenting with potential substance misuse risk factors were identified from diagnosis codes and filtered for specific substance misuse concerns. Highly suspected cases were then audited for management and discharge outcome at point of departure from the department.

A total of 334 young persons between the age of 9-17 years presented to our A\&E Department between December 2011-January 2012. Forty (12\%) had diagnosis coding for alcohol intoxication, alcohol withdrawal, deliberate drug overdose, head injury, alleged assault, faint, road traffic accident injuries undetermined, psychiatric problem, hyperventilation, collapse and injury to face. Of these, $9(22.5 \%)$ were young persons between $15-17$ years-old and identified as high risk for substance misuse. Only 1 case was referred to the Adult medical team, and was admitted, but none of the remaining patients were referred to a Paediatric team and were discharged home or had absconded. Only 1 patient had a documented use of a "Substance Misuse Assessment Tool", and none had Psychiatric or CAMHS input nor were referred to a Young Person-specific Substance Misuse service.

Health professionals who regularly manage young people in A\&E, including A\&E nursing staff need essential training in assessing Young People for Substance Misuse. Young Person-specific Substance Misuse clinical guidelines would be useful to increase case management confidence for Paediatricians, junior A\&E doctors and Adult Physicians. A valuable resource to the NHS, referral to Young Person-specific services in Substance Misuse should be considered in these guidelines. Multi-departmental, multi-disciplinary agreement is imperative for successful implementation. 